\title{
ASTEROID VERSUS COMET DISCRIMINATION FROM ORBITAL DATA
}

\author{
L. KRESAK
}

The orbital comet-asteroid criteria, their premises, dynamical reasoning, and ranges of applicability are reviewed. Mapping of all known comets and asteroids in a plane of energy intergral in the two body problem (1/a) vs. that in the restricted three-body problem (Tisserand invariant) is presented. The potential evolutionary paths from different sources of active comets into shortperiod orbits are delineated and interfaced with the process of reducing the perihelion distances of the asteroids. The significance of resonances with Jupiter is emphasized. Statistics of observed close approaches of individual comets and asteroids to the Earth is analyzed to estimate their relative fluxes. Active cometary nuclei are found to represent about $1 / 8$ of the flux of objects with radii exceeding $1 \mathrm{~km}$ in the vicinity of the Earth, and their contribution tends to diminish significantly for still smaller bodies. However, there is no evidence against comets leaving inactive asteroid-like nuclei with considerable 1 ifetimes which may represent a significant, though secondary, source of meteors and even meteor streams. An overwhelming majority of the Apollo and Amor objects are suggested to be of asteroidal nature; the most probable exceptions are selected and recommended for detailed observation.

The comets and the asteroids represent two basic types of interplanetary bodies differing in composition, structure, place of origin, and evolutionary history. Their simplest discrimination is by appearance which is always stellar for the asteroids (objects of apparent diameters from 0!.001 to $1^{\prime \prime}$ shining in reflected sunlight) and nebulous for the comets developing large atmospheres, and often also tails, composed of gas, plasma, dust and ice grains. However, this characteristic feature is time-1imited: it only appears when the comet is near enough to the Sun, and eventually has to vanish when all its supply of volatiles has been exhausted. Since the sunlight reflected from the solid nucleus of an active comet is only a small fraction of its total light, the brightness has to decrease drastically when the comet becomes inactive, making its discovery conditions most difficult. At present we know with confidence on $1 y$ one extinct comet nucleus -- denoted as asteroid 944 Hidalgo. A few short-period comets, in particular P/Arend-Rigaux and P.Neujmin 1, looked perfectly asteroidal during some returns to the Sun but developed diffuse halos during others. It may be noted that the central condensation in the coma is sometimes so small that it may be mistaken for an asteroidal object on photographs. In fact, several new comets were announced and provisionally designated as asteroids (Comet 1917 I I $=1916 \mathrm{ZK}, 1939 \mathrm{IV}=1939 \mathrm{CB}, 1942 \mathrm{II}=1942 \mathrm{EA}$ ). 
The discrimination between comets and asteroids becomes essential during the last phases of evolution of comets where two areas of puzzling problems stand out :

(1) What is the ultimate fate of comets? Do they disintegrate completely or do they, after the depletion of volatiles, leave compact nuclei; and if so, how can these be distinguished from small asteroids?

(2) What is the origin of meteoroids of different sizes? Can the products of disintegration of comets be distinguished from the fragments of asteroids using the orbital data alone?

The latter problem is complicated by the fact that the motion of small particles is also perturbed by effects other than gravitation, especially by direct radiation pressure and poynting-Robertson effect. This aspect is sometimes overemphasized, however, because it is overlooked that the lifetimes of meteor particles are short. A considerable proportion of observed meteors come from currently active comets, and their orbits do not reveal observable deviations from the parent comets in the required sense (Kresáková 1974). There are meteor streams whose parent bodies are unknown but their structure suggests that they must have formed near their present orbits. A very significant case is the wellknown Geminid stream the orbit of which resembles asteroid Icarus rather than any comet. Thus the problem of comet-asteroid discrimination is relevant to meteor astronomy as well.

The instantaneous motion of a given body is commonly expressed by a set of six osculating elements defining a Keplerian, or unperturbed, orbit around the Sun. These elements are continuously changing due to the perturbations, and the existence of a strict comet/asteroid criterion would require the presence of a dividing surface in the phase-space of some orbital elements which would separate the two types of bodies and could not be crossed during their evolution. For meteor orbits, restricted by the earth-crossing condition, two empirical criteria of this kind were proposed by Whipple (1954) and Kresák (1967). Both define functionally a dividing curve in the a/e plane and are based solely on the distribution of known comets and asteroids in this plane. A simpler criterion of broader use is that of aphelion distance. This is equivalent to the statement that orbits of the asteroids are confined to the sphere with a diameter equal to the heliocentric distance of Jupiter.

A11 these criteria have a common drawback in considering only the geometry of the orbit, and apparently fail for resonant motions. The essence of the difference is whether the body approaches Jupiter, and not whether its orbit does so. A well-known example of resonant orbits avoiding encounters with Jupiter, which would be classified as cometary basing on the aphelion distance alone is the Trojan asteroids. The importance of virtual encounters with Jupiter was stressed by Marsden $(1970,1971)$ who pointed out a most important circumstance: just those comets which look nearly asteroidal tend to avoid Jupiter by librating motions.

Figure 1 shows the apparent similarity of the orbits of some comets and asteroids. The upper series illustrates the effects of resonance. The asteroid of the first pair, 1036 Ganymedes, is not far from the resonance of $3 / 1$ with the motion of Jupiter but does not librate in longitude; however, a coupling of the perturbations in inclination and eccentricity prevents closer approaches to Jupiter (Kozai 1962). The asteroid of the second pair, 1362 Griqua, librates around the $2 / 1$ resonance so that it never passes aphelion when Jupiter is in this part of its orbit. The similarity of the third pair is most striking, the orbit of Comet Oterma being entirely indistinguishable from the librating asteroids of the Hilda group. But just in this case the actual motion is drastically different. While the motion of the Hilda asteroids is stabilized in the $3 / 2$ resonance and avoids Jupiter, the comet was in phase with Jupiter for three revolutions between two close approaches. It was first captured into a short- 

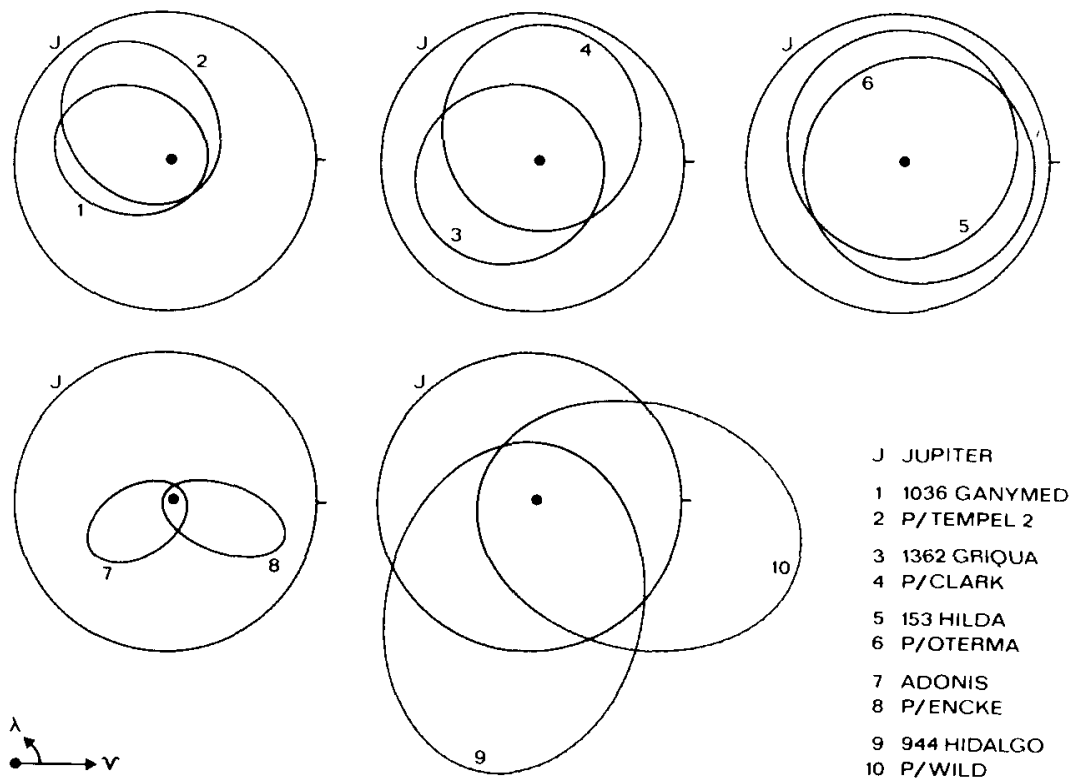

$J$ JUPITER

1 1036 GANYMEDES

2 P/TEMPEL 2

3 1362 GRIOUA

4 P/CLARK

5 153 HILDA

6 P/OTERMA

7 AOONIS

8 P/ENCKE

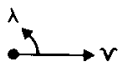

9944 MIDALGO

$10 \mathrm{P} /$ WILD

Figure 1. Apparent similarity between orbits of selected comet-and-asteroid pairs.

period orbit and at the next encounter ejected again. The next pair consists of the earth-crossing asteroid Adonis and Comet Encke which is unique by its small aphelion distance. If its nongravitational deceleration continues the shape of its orbit will become even more alike that of Adonis. The last pair is, in all probability, a pair of comets at different stages of physical evolution. It appears almost certain tht asteroid 944 Hidalgo is in fact an extinct cometary nucleus.

The limited accuracy of the osculating elements obtained from positional observations and the presence of nongravitational forces make it impossible to trace the motion of comets backwards over more than one close approach to Jupiter (i.e., more than a few centuries, as a rule). For this reason the past evolution of individual real objects is essentially indeterminate. Relevant information can only be obtained from model computations of randomly selected sets of orbits -- a procedure most successfully applied in a series of papers by Everhart (1968, $1969,1972,1973 \mathrm{ab})$ or by using an approximation by the restricted three-body problem. The latter approach, already applied by a number of authors (e.g., Opik 1963, Rabe 1971 and 1974; Everhart 1972; Vaghi 1973 ab; Lowrey 1973; Whipple 1976, Kresák $1969,1972 \mathrm{ab}, 1973$ ) will be used here. The approximation is substantiated by the controlling influence of Jupiter during the critical phases of evolution and by quantitative comparison with other secondary effects (Kresák 1972b). However, since an adequate stability of the decisive parameter -- the Jacobi constant or Tisserand invariant -- has been questioned by Everhart (1976) reference to his results will often be made.

Figure 2 shows the energy integral in the two-body problem, $a^{-1}$, plotted against the energy integral in the rotating frame of the restricted circular three-body problem Sun/Jupiter/comet or asteroid, T. Both quantities are in the same units, $\mathrm{AU}^{-1}$, so that the values of $\mathrm{T}$ should be multiplied by the semimajor axis of Jupiter, 5.2, to convert them into the canonical units used in most 


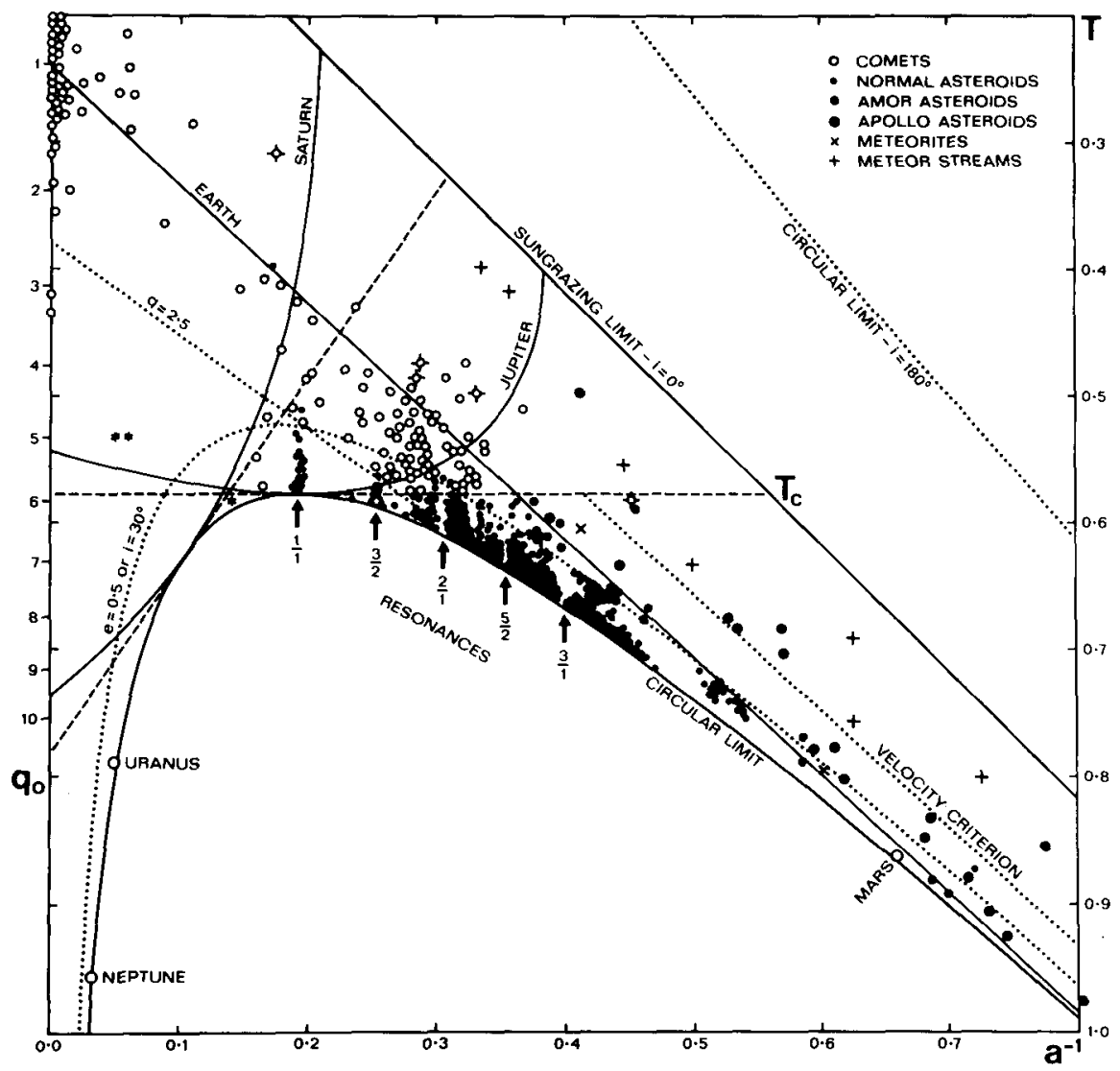

Figure 2. Orbital energy in the 2-body problem $a^{-1}$, versus the energy in the rotating frame of the restricted three-body problem $T$ with the sun and Jupiter. Both energies are per unit mass and expressed in $A U^{-1}$.

papers. Conservation of energy requires the evolutionary paths of smaller bodies perturbed by Jupiter to follow a horizontal direction in this diagram, with long periods spent relatively at rest and jumps experienced during encounters with Jupiter. On these occasions, the terms in the Jacobi integral containing the mass ratio Jupiter/Sun and reciprocal distances neglected in the Tisserand invariant, may become appreciable and make the body deviate from the horizontal. But when the approach is over and the change of the orbit is completed the body assumes the original value of $\mathrm{T}$ at the new value of $\mathrm{a}^{-1}$. An analysis of a number of encounters has shown that the changes in $T$ are, on the average, $5 \%$ of those in $a^{-1}$ for time-spans of centuries, so that the scatter of the evolutionary paths from the horizontal is some $3^{\circ}$ (Kresák 1972b). This is mainly due to the eccentricity of Jupiter's orbit. A capture into a short-period orbit is represented by a major displacement to the right, an ejection from the solar system by a displacement beyond the left edge of the diagram. Libration can occur at simple resonances with the mean motion of Jupiter and results in small periodic displacements with characteristic periods of $100-500$ years. In the case of locked libration the mean position may remain the same practically forever. 
All known short-period comets (elements from Marsden 1975). numbered asteroids (elements from Chebotarev 1975 and MPC), and all known earth-crossing asteroids are mapped in the diagram*. The potential regions of their origin are 10cated as follows. The main asteroid belt divided by the resonance gaps is seen in the middle, with the Hilda zone and the Trojan Clouds on its left. The Oort Cloud of comets is centered at $\mathrm{a}^{-1} \cong 0, \mathrm{~T} \cong 0$ and runs along the left edge of the diagram. Only its low-inclination large-q tail comprising about $10 \%$ of known comets is depicted; the left-hand scale indicates the minimum perihelion distance corresponding to zero inclination, $q_{0}$. Al alternate primary source of short-period comets suggested by Whipple (1964) is a circular belt near the orbit of Neptune running close to the circular limit on lower left. Suggested secondary sources are: the region of intermediate-period comets of low inclination and perihelia near Jupiter (Delsemme 1973; double asterisk), a belt of nearly circular orbits between Jupiter and Saturn (Kresak 1972a; asterisk), and the Trojan Clouds (Rahe 1971 and 1974).

One essential property of the diagram is that the evolutionary paths due to perturbations by other planets also appear as parallel straight lines, and the corresponding displacements add to those by Jupiter. Provided that the inclination is low the region of possible encounters with individual planets are delimited as shown by the full curves drawn for Jupiter, Saturn, and the Earth. Perturbations are most frequent and most efficient near the boundary, and the displacements are parallel to the tangent of this boundary at the point of maximum perturbation, or satellite position, corresponding to the elements of the planet (dashed lines).

Another point of importance is the existence of the circular limit, a barrier below which no real orbits exist. Thus if a comet is started in a lowinclination orbit far beyond Jupiter (say, $q>10$ ) then, irrespective of its eccentricity, the Tisserand invariant with respect to Jupiter is high. Multiple capture by the outer planets, as suggested and investigated by KazimirchakPolonskaya $(1967,1971,1972)$ may occur. But this will force the comet to climb up the barrier, and a capture into a short-period orbit will become possible until after $\mathrm{T}$ has been reduced to approximately the critical value $\mathrm{T}_{\mathrm{C}}=0.58$. This process is well illustrated by the example used by Everhart (1976) to show the instability of the Tisserand invariant. However, his arguments do not invalidate the use of the three-body approximation for the capture problem. It can be nicely seen from his figure that the same critical value of $T$ is restored whenever the perihelion of the comet approaches the orbit of Jupiter thus rendering a capture into a short-period orbit possible.

The evolution is most straightforward and therefore easiest for those comets which have nearly a critical value of $T$ when entering the region of Jupiter for the first time. This is just the "capture region" found empirically by Everhart (1972) in his numerical experiments. Everhart (1976) also noted that comets coming from this source cannot be distinguished by orbital characteristics from those started in nearly circular orbits near Jupiter. This is an obvious consequence of the fact that the two evolutionary paths converge at the top of the barrier where also the comets of high original eccentricity would pass a stage of nearly circular orbits. As a rule, this happens before the comets become observable. The conventional limit of detectability of shortperiod comets $(\mathrm{q}=2.5$, $\mathrm{i}$ small) is marked by a dotted line.

If we proceed further up in the diagram the capture becomes less probable due to an increasing jovicentric velocity at the point of encounter, and the necessity of a favourable position of nodes. At the same time the sungrazing limit becomes important inasmuch as the low-inclination orbits would reduce their perihelion distances and age very rapidly before their aphelion approaches

\footnotetext{
* A few comets of the type of Comet halley lie above the upper edge; 1566 rcarus, 1865 Cerberus and $1976 \mathrm{AA}$ fall outside the diagram on its Iower right; the dot outside the margin is 1620 Geographos.
} 
Jupiter. This is why Everhart (1972) in his numerical experiments could not find short-period orbits originating from nearly parabolic orbits of small perihelion distance or high inclination.

It may be concluded that all comets entering short-period orbits have their Tisserand invariant smaller than, and most close to, the critical value $T_{c}=$ 0.58. On the other hand, the invariant is larger than this for all non-librating asteroids except 944 Hidalgo and $1973 \mathrm{NA}$. The librating asteroids are bound to small oscillations around a fixed point of the diagram and cannot enter cometlike orbits unless the resonance is destroyed. An escape from the resonance can effectively be triggered by the momentum imparted by collision and hence is of special importance for asteroidal fragments observable as meteorites, as shown by Zimmerman and Wetherill (1973) for the case of $2 / 1$ resonance. Similar investigations appear desirable for resonances of $1 / 1,3 / 2$ and $5 / 2$. It is interesting that the aphelia of some Amor asteroids tend to cluster near the mean radius of the $3 / 2$ resonance, $Q=4.0$. There is also some clustering near the Tisserand invariant of Mars, and it can be reasonably assumed that the respective orbits have been derived from close approaches to that planet. Since dying comets can be captured into resonance with the aid of nongravitational accelerations the resonance islands in the diagram represent potential regions of exchange from "cometary" to "asteroidal" orbital characteristics and vice versa.

Admittedly, our criterion breaks down if both the aphelion and perihelion distances become so small that the inner planets can take over the controlling influence from Jupiter. The case of 1685 Toro, locked into libration with Earth and Venus (Williams and Wetherill 1973) shows that this is possible. On the other hand, the case of $\mathrm{P} /$ Encke demonstrates that active short-period comets in retrograde rotation can reduce their aphelion distances to $4 \mathrm{AU}$ at least. Finally, we cannot disregard the possibility that some earth-approaching asteroids are remnants of an original, much more abundant population in the region of terrestrial planets. Their orbits are rather stable on shorter time-scales (Chebotarev et al. 1972) and their collisional lifetimes are not so short that a fraction could not survive (Öpik 1963; Wetherill and Williams 1968; Weidenschilling 1973).

It appears that a great majority of the earth-approaching small asteroids are really of asteroidal origin. Their prevalence of $4: 1$, as estimated by Whipple (1973) seems to be a conservative lower limit. The question remains how the ex-comets can be distinguished from the rest. Four relevant criteria have already been suggested: (1) a higher geocentric velocity (Anders and Arnold 1965; Anders and Mellick 1971; Lowrey 1973; this criterion is also indicated in Figure 2); (2) orbital association with minor meteor showers (Sekanina 1973;) (3) possibility of reduction of the aphelion distance under certain assumptions on the nongravitational effects (Sekanina 1971); and (4) flat lightcurve pointing to a nearly spherical shape (Gehrels 1972). Classifications according to (1) and (2) are in good correlation, however, this must be taken with reserve because the detection limit of meteors depends strongiy on velocity. Criterion (3) concerns just those objects for which explanation in terms of ejection of asteroids from resonance appears plausible. As regards (4), we cannot be sure that all cometary nuclei are nearly spherical: in fact, their frequent splitting may be associated with irregular wasting of the material (Whipple and Huebner 1976) leading to rotational instability of irregularly shaped bodies.

All these criteria agree in 1936 CA Adonis being a first-rank candidate for cometary origin, however in this case three recent discoveries with similar dynamical characteristics should be added: 1866 Sisyphus, $1973 \mathrm{EA}$, and $1974 \mathrm{MA}$. The Tisserand invariant points clearly to 944 Hidalgo and 1973 NA being excomets, with a marginal case of 1580 Betulia. Recoveries and precise observa- 
tions of these objects with modern astrophysical techniques would be highly desirable. Such observations should be extended to some librating asteroids, like 1362 Griqua or $1922=1949 \mathrm{HC}$, as it appears that dying comets tend to settle in resonant orbits (Marsden 1971; Franklin et al. 1975).

As the number of known earth-approaching asteroids is growing rapidly, it is now possible to gather some statistical information about the relative flux of different types of larger interplanetary bodies in the earth environment. An analysis of this problem will be published elsewhere (Kresák 1977) but some of the conclusions can be summarized as follows:

About 40 objects with radii exceeding $1 \mathrm{~km}$ approach the Earth within 0.1 AU every century. 30 of them are Apollo asteroids ( $q<1$ ), five Amor asteroids ( $q>1)$, two active short-period comets $(P<20 \mathrm{yr}$ ), and three active comets of internediate or long period $(P>20 \mathrm{yr})$. While the sizes of the comets are rather uncertain and some objects from below the limit may be included, it is most interesting that their present statistics.seem to be essentially complete, in particular for the objects of longer period. There are evidently no small active comets, and extrapolation of the observed numbers using exponential incremental laws is clearly misleading. There are no known asteroidal objects moving in long-period orbits. Among the meteors only this type of orbit can be distinguished quite unambiguously; it comprises $26 \%$ of photographic meteors but less than 5\% of their mass (based on Jacchia and Whipple 1961). For the other groups the discrimination is much more difficult but a relative lack of correlation between physical characteristics of meteors and their orbits suggests a cometary origin for a great majority of them (Jacchia et al. 1967). On the other hand, there is $1 \mathrm{ittle}$ difference between the orbits of some meteors and the meteorites which are apparently of asteroidal origin. It is important to note that neither a majority of meteors nor the bona fide asteroids except Hidalgo move in orbits typical for active comets. The missing link is the orbital evolution of dying cometary nuclei.

There is little doubt that below the limit of $1 \mathrm{~km}$ radius the prevalence of asteroidal objects over the cometary ones increases. Even so the large disproportion between active and inactive objects passing near Earth still permits the assumption that comets do leave small extinct nuclei the lifetimes of which are long compared with their active evolutionary phases. These cometary remnants may represent a significant source of sporadic meteoroids, and their sudden fragmentation by collision may even produce meteor streams. But their rate of release of meteoroids must be much lower than that of active comets.

\section{REFERENCES}

Anders, E., and Arnold, J. R. 1965, Science, 149, 1494.

Anders, E., and Mellick, P. J. 1969, in Meteorite Research, ed. P. M. Millman, Astrophys. Space Sci. Libr. 12, Reidel, Dordrecht, p. 559.

Chebotarev, G. A., ed. 1975, Efemeridy Malykh Planet 1976, Nauka, Leningrad.

Chebotarev, G. A., Belyaev, N. A., and Eremenko, R. P. 1972, in The Motion, Evolution of Orbits, and Origin of Comets, IAU Symp. 45, p. 431.

Delsemme, A. H. 1973, Astron. Astrophys., 29, 377.

Everhart, E. 1968, Astron. J., 73, 1039.

Everhart, E. 1969, Astron. J., 74, 735.

Everhart, E. 1969, Astrophys. Letters, 10, 131.

Everhart, E. 1973a, Astron. J., 78, 316.

Everhart, E. 1973b, Astron. J., 78, 329.

Everhart, E. 1976, in The Study of Comets, IAU Co11. $25=$ NASA SP-393, p. 445 .

Frank1in, F. A., Marsden, B. G., Williams, J. G., and Bardwe11, C. M. 1975, Astron. J., 80, 729 . 
Gehrels, T. 1972, in From Plasma to Planet, Nobe1 Symp., Almquist and Wicksell, Stockholm, p. 169.

Jacchia, L. G., and Whipp1e, F. L. 1961, Smithson. Contr. Astrophys. 4., 97.

Jacchia, L. G., Verniani, F., and Briggs, R. E. 1967, Smithson. Contr. Astrophys., 10,1.

Kazimirchak-Polonskaya, E. I. 1967, Astron. Zh. 44, 439.

Kazimirchak-Polonskaya, E. I. 1971, Byul. Inst. Teor. Astron. Leningrad, 12, 796.

Kazimirchak-Polonskaya, E. I. 1972, in The Motion, Evolution of Orbits, and origin of Comets, IAU Symp. 45, p. 373.

Kozai, Y. 1962, Astron. J., 67, 591 .

Kresák, L. 1967, in Meteor Orbits and Dust, Smithson. Contr. Astrophys., 11, NASA SP-135, p. 9 .

Kresák, L. 1969, Bull. Astron. Inst. Czech., 20, 177.

Kresák, L. 1972a, in The Motion, Evolution of Orbits, and Origin of Comets, IAU Symp. 45, p. 503.

Kresák, L. 1972b, Bull. Astron. Inst. Czech., 23, 1.

Kresák, L. 1973, in Evolutionary and Physical Properties of Meteoroids, IAU Co11. 13, NASA SP-319, p. 331.

Kresák, L. 1977, Bull. Astron. Inst. Czech., 28, in print.

Kresáková, M. 1974, Bull. Astron. Inst. Czech., 25, 191.

Lowrey, B. E. 1973, Astron. J., 78, 428.

Marsden, B. G. 1970, in Periodic Orbit, stability and Resonances, ed. G.E.0. Giacaglia, Reide1, Dordrecht, p. 151 .

Marsden, B. G. 1971, in Physical studies of Minor Planets, IAU Col1. 12, NASA $\mathrm{SP}-267, \mathrm{p} .413$.

Marsden, B. G. 1975, Catalogue of Cometary orbits, Centr. Bur. Telegr. Astron., Cambridge, Mass.

Oort, J.H. 1950, Bull. Astron. Inst. Netherlands, 11, 91.

Öpik, E. J. 1963, Adv. Astron. Astrophys., 2, 219.

Öpik, E. J. 1973, Astrophys. Space. Sci., 21, 307.

Rabe, E. 1971, in Physical Studies of Minor Planets, IAU Coll. 12, NASA SP-267, p. 407

Rabe, E. 1974, in Asteroids, Comets, Meteoric Matter, IAU Col1. 22, Editura Acad. Bucharest, p. 165.

Sekanina, Z. 1971, in Physical studies of Minor Planets, IAU Col1. 12, NASA SP-267, p. 423 .

Sekanina, Z. 1973, Icarus, 18, 253.

Sitarski, G. 1968, Acta Astron. 18, 171 .

Vaghi, S. 1973a, Astron. Astrophys., 24, 107.

Vaghi, S. 1973b, Astron. Astrophys., 29, 85.

Weidenschilling, S. J. 1975, Astron. J., 80, 145.

Wetheril1, G. W., and Williams, J. G. 1968, J. Geophys. Res., 73, 635.

Whipple, F. L. 1954, Astron. J., 59, 201.

Whipple, F. L. 1964, Proc. Nat. Acad. Sci., 51, 711.

Whipple, F. L. 1973, The Moon, 8, 340 .

Whipple, F. L. 1976, Icarus, in print.

Whipple, F. L., and Huebner, W. F. 1976, Ann. Rev. Astron. Astrophys., in print. Williams, J. G., and Wetherill, G. W. 1973, Astron. J., 78, 510.

Zimmerman, P. D., and Wetherill, G.W. 1973, Science, 182, 51 .

\section{DISCUSSION}

WETHERILL: You have made a very good summary of a complex subject. There is one very minor point which you mentioned which I think can be clarified. It can be concluded that no significant number of present Apollo objects are remnants 


\section{ASTEROIO-COMET DISCRIMINATION}

of a primordial earth-crossing population for two reasons: 1) The dynamic lifetime of these bodies is about 20 - 30 million years, shorter than the value given by opik. If remnants still exist, this would require an unacceptable large initial mass $210^{60} \mathrm{~g}$. 2) Any significantly larger number in the last $3.5 \times 10^{9}$ yrs. would be in conflict with the lunar and terrestrial cratering record, which indicates a nearly constant rate since the mare surfaces were formed.

KRESAK: This is certainly true for a great majority of the original population. However, there seems to be a possibility of exceptionally long lifetimes connected with the storage of some asteroids in resonant orbits. With the aid of nongravitational effects (collisions, jet effects on active comets), and perturbations by other planets, libration can be both triggered and destroyed. The transitions between librating and circulating motion appear to be most important for the survival times, and also for the changes from "cometary" to "asteroidal" orbits and vice versa. I agree that the outer regions of the asteroid belt seem to be a more suitable place for a long-term storage of objects which later appear as Apollos. The small aphelion distances of some of them (1976 AA!) would then point to a capture by the Earth. 